\title{
Polarity in signed symmetric group and signed transformation semigroup
}

\author{
${ }^{1}$ Mogbonju, M.M., ${ }^{2}$ Ogunleke, I.A., ${ }^{3}$ Adeniji, A.O. \\ ${ }^{1,2}$ Faculty of Science, Department of Mathematics, P.M.B.117. University of Abuja F.C.T. Nigeria \\ ${ }^{3}$ Alvan IkokuFedereal College of Education. Department of Mathematics P.M.B. 1033 Owerri, Imo State Nigeria \\ mmogbonju@gmail.com.
}

\begin{abstract}
Let $P S S_{n}, P S T_{n}$ and $P S S i n g_{n}$ be polarity of symmetric group, polarity of signed transformation semigroup and polarity of signed singular mapping respectively from $X_{n} \rightarrow X_{n}^{*}$ where $X_{n}=\{1,2,3, \cdots, n\}$ and $X_{n}^{*}=$ $\{-n, \cdots,-3,-2 .-1.0,1,2,3, \cdots, n\}$, and $X_{n} \subset X_{n}^{*}$. The aim of thus paper is to determine the order of $P S S_{n}$, $\operatorname{PST}_{n}$ and $\operatorname{PSSing}_{n}$.
\end{abstract}

Keywords:polarity, semigroup, signed symmetric group, signed transformation semigroup, signed singular mapping

DOI: $10.7176 / \mathrm{MTM} / 9-7-03$

Publication date: July $31^{\text {st }} 2019$

\section{Introduction}

The study of symmetric groups, alternating groups and dihedral groups has made a significant contribution to group theory, so has the study of various subsemigroups of $T_{n}, P_{n}$ and $I_{n}$ (Bashar 2010; Howie 2002; Laradji and Umar 2004; Umar 1992). Extensive research work have been done in the area of semigroup see Higgins 1992),(Laradji and Umar 2004), (Adeniji2012), (Adeshola2013). (Bakare and Makanjuola 2013), (Mogbonju2015).(James and Kerber1981) defined permutation group on set $X_{n} \rightarrow X_{n}^{*}$ and $S T_{n}$ (the signed transformation group) is a semigroup analogue of $T_{n}$ and $S S_{n}$ (signed symmetric group) is the units of $S_{n}$. However (Mogbonj2015) studied signed transformation semigroup of full partial and partial $1-1$. He defined a signed transformation as the set of all mapping from $\alpha$ : $\operatorname{dom}(\alpha) \subseteq X_{n} \rightarrow \operatorname{Im}(\alpha) \subset X_{n}^{*}$ where $X_{n}=$ $\{1,2,3, \cdots, n\}$ and $X_{n}^{*}=\{-n, \cdots,-3,-2 .-1.0,1,2,3, \cdots, n\}$. Dom $(\alpha)$ stands for the domain of $\alpha$ while $\operatorname{lm}(\alpha)$ as lmage of $\alpha$. (Mogbonju2015) studied the order, idempotent, nilpotent and the chain decomposition of of full and patial transformation semigroup. Also (Mogbonju2019) studied the polarity in signed order preserving, order decreasing and both order preserving and order decreasing semigroup respectively.

The semigroup of singular self-mapping of $X_{n}$ was defined by Howie(1996) as $\operatorname{Sing}_{n}=T_{n} \backslash S_{n}=$ $\left\{\alpha \in T_{n}: \operatorname{lm}(\alpha) \leq n\right\}$.(Mogbonju2019) also studied the order, idempotent and chain decomposition of signed singular mappings semigroup on a set $\alpha: \operatorname{dom}(\alpha) \subseteq X_{n} \rightarrow \operatorname{Im}(\alpha) \subset X_{n}^{*}$.

The following known results and theorem from (Mogbonju2015) are very crucial to this work.

Theorem 1.1[Mogbonju (2015)]Theorem 3.2.1]. Let $S=S S_{n}$ and if $\alpha \in S S_{n}, n \geq 1$ then $|S|=2 n !-$ $\left(2^{n}-2\right) n$ !

Theorem 1.2[Mogbonju (2015)]Theorem 3.2.2]. Let $S=S T_{n}$, then $|S|=2 n^{n}+n^{n}\left(2^{n}-2\right)$

Theorem 1.3[Mogbonju (2015)]Theorem 3.6.1]. Let $S=\operatorname{Sing}_{n}$ if $\alpha \in S, n \geq 0$, then $|S|=2^{n}\left(n^{n}-n !\right)$

Theorem 1.4[Mogbonju (2015)]Theorem 3.6.1]. Let $S=S P T_{n} \backslash S S_{n}$ then $|S|=(2 n+1)^{n}-2^{n} n$ ! 


\section{Methodology}

Let $P S S_{n}, P S T_{n}$ and $P S \operatorname{Sing}_{n}$ be polarity of signed symmetric group, polarity of signed transformation semigroup and polarity of signed singular mapping defined on a set $\alpha: X_{n} \rightarrow X_{n}^{*}$ where $\alpha: \operatorname{dom}(\alpha) \subseteq X_{n} \rightarrow \operatorname{Im}(\alpha) \subset X_{n}^{*}$

\subsection{Elements in $S S_{n}, S T_{n}$ and $\operatorname{SSing}_{n}$}

The set of elements in $S S_{2}$ is as follows:

$$
\left|S S_{2}\right|=\left\{\left(\begin{array}{cc}
1 & 2 \\
-1 & -2
\end{array}\right),\left(\begin{array}{cc}
1 & 2 \\
-2 & -1
\end{array}\right),\left(\begin{array}{cc}
1 & 2 \\
-1 & 2
\end{array}\right),\left(\begin{array}{cc}
1 & 2 \\
-2 & 1
\end{array}\right),\left(\begin{array}{cc}
1 & 2 \\
1 & -2
\end{array}\right),\left(\begin{array}{cc}
1 & 2 \\
2 & -1
\end{array}\right),\left(\begin{array}{cc}
1 & 2 \\
1 & 2
\end{array}\right),\left(\begin{array}{ll}
1 & 2 \\
2 & 1
\end{array}\right)=8\right\}
$$

The set of elements in $S T_{2}$ is as follows:

$$
\left|S T_{2}\right|=\left\{\begin{array}{r}
\left(\begin{array}{cc}
1 & 2 \\
1 & -1
\end{array}\right),\left(\begin{array}{cc}
1 & 2 \\
-1 & 1
\end{array}\right),\left(\begin{array}{cc}
1 & 2 \\
-1 & -1
\end{array}\right),\left(\begin{array}{cc}
1 & 2 \\
1 & -2
\end{array}\right),\left(\begin{array}{cc}
1 & 2 \\
-1 & 2
\end{array}\right),\left(\begin{array}{cc}
1 & 2 \\
-1 & -2
\end{array}\right),\left(\begin{array}{cc}
1 & 2 \\
2 & -1
\end{array}\right),\left(\begin{array}{cc}
1 & 2 \\
-2 & 1
\end{array}\right), \\
\left(\begin{array}{cc}
1 & 2 \\
-2 & -1
\end{array}\right),\left(\begin{array}{cc}
1 & 2 \\
2 & -2
\end{array}\right),\left(\begin{array}{cc}
1 & 2 \\
-2 & 2
\end{array}\right),\left(\begin{array}{cc}
1 & 2 \\
2 & -2
\end{array}\right),\left(\begin{array}{ll}
1 & 2 \\
1 & 1
\end{array}\right),\left(\begin{array}{ll}
1 & 2 \\
1 & 2
\end{array}\right)\left(\begin{array}{ll}
1 & 2 \\
2 & 1
\end{array}\right),\left(\begin{array}{ll}
1 & 2 \\
2 & 2
\end{array}\right)
\end{array}\right\}=16
$$

The set of elements in SSing $_{2}$ is as follows:

$$
\left|\operatorname{SSing}_{2}\right|=\left\{\left(\begin{array}{cc}
1 & 2 \\
-1 & -1
\end{array}\right),\left(\begin{array}{cc}
1 & 2 \\
-2 & -2
\end{array}\right),\left(\begin{array}{cc}
1 & 2 \\
-1 & 1
\end{array}\right),\left(\begin{array}{cc}
1 & 2 \\
-2 & 2
\end{array}\right),\left(\begin{array}{cc}
1 & 2 \\
1 & -1
\end{array}\right),\left(\begin{array}{cc}
1 & 2 \\
2 & -2
\end{array}\right),\left(\begin{array}{cc}
1 & 2 \\
1 & 1
\end{array}\right),\left(\begin{array}{ll}
1 & 2 \\
2 & 2
\end{array}\right)\right\}=8
$$

\subsection{Polarity of elements in $S S_{n}, S T_{n}$ and $S S_{i n g}$}

Polarity of elements in $S S_{n}$ is as follows:

When $n=1$

$$
\left|P S S_{1}\right|=\left\{\left(\begin{array}{c}
1 \\
-1
\end{array}\right)\right\}=1
$$

When $n=2$

$$
\left|P S S_{2}\right|=\left\{\left(\begin{array}{cc}
1 & 2 \\
-1 & 2
\end{array}\right),\left(\begin{array}{cc}
1 & 2 \\
1 & -2
\end{array}\right),\left(\begin{array}{cc}
1 & 2 \\
-1 & -2
\end{array}\right),\left(\begin{array}{cc}
1 & 2 \\
-2 & 1
\end{array}\right),\left(\begin{array}{cc}
1 & 2 \\
2 & -1
\end{array}\right),\left(\begin{array}{cc}
1 & 2 \\
-2 & -1
\end{array}\right)\right\}=6
$$

Image of element in $\mathrm{PSS}_{2}$

$$
\begin{gathered}
\left|\operatorname{Im}\left(\alpha^{-}\right)\right|=\left\{\left(\begin{array}{cc}
1 & 2 \\
-1 & -2
\end{array}\right),\left(\begin{array}{cc}
1 & 2 \\
-2 & -1
\end{array}\right)\right\}=2 \\
\left|\operatorname{Im}\left(\alpha^{*}\right)\right|=\left\{\left(\begin{array}{cc}
1 & 2 \\
-2 & 1
\end{array}\right),\left(\begin{array}{cc}
1 & 2 \\
-1 & 2
\end{array}\right),\left(\begin{array}{cc}
1 & 2 \\
1 & -2
\end{array}\right),\left(\begin{array}{cc}
1 & 2 \\
2 & -1
\end{array}\right)\right\}=4
\end{gathered}
$$

Table 2.1: Values of elements in $P S S_{n}$

\begin{tabular}{|c|c|c|c|}
\hline$n$ & $\left|\operatorname{Im}\left(\alpha^{-}\right)\right|$ & $\left|\operatorname{Im}\left(\alpha^{*}\right)\right|$ & $\left|P S S_{n}\right|=n !+\left(2^{n}-n\right) n !$ \\
\hline 1 & 1 & - & 6 \\
\hline 2 & 2 & 4 & 42 \\
\hline 3 & 6 & 36 & 360 \\
\hline 4 & 24 & 336 & 3420 \\
\hline 5 & 120 & 3300 & 45360 \\
\hline 6 & 720 & 44640 & \\
\hline
\end{tabular}


Polarity of elements in $S T_{n}$ is as follows:

When $n=1$

$$
\left|P T S_{1}\right|=\left\{\left(\begin{array}{c}
1 \\
-1
\end{array}\right)\right\}=1
$$

When $n=2$

$$
\left|P T S_{2}\right|=\left\{\begin{array}{r}
\left(\begin{array}{cc}
1 & 2 \\
-1 & -1
\end{array}\right),\left(\begin{array}{cc}
1 & 2 \\
-1 & -2
\end{array}\right),\left(\begin{array}{cc}
1 & 2 \\
-2 & -1
\end{array}\right),\left(\begin{array}{cc}
1 & 2 \\
-2 & -2
\end{array}\right),\left(\begin{array}{cc}
1 & 2 \\
1 & -1
\end{array}\right),\left(\begin{array}{cc}
1 & 2 \\
-1 & 1
\end{array}\right) \cdot \\
\left(\begin{array}{cc}
1 & 2 \\
1 & -2
\end{array}\right),\left(\begin{array}{cc}
1 & 2 \\
-1 & 2
\end{array}\right),\left(\begin{array}{cc}
1 & 2 \\
2 & -1
\end{array}\right),\left(\begin{array}{cc}
1 & 2 \\
-2 & 1
\end{array}\right),\left(\begin{array}{cc}
1 & 2 \\
2 & -2
\end{array}\right),\left(\begin{array}{cc}
1 & 2 \\
-2 & 2
\end{array}\right),
\end{array}\right\}=8
$$

Image of element in $\mathrm{PSS}_{2}$

$$
\begin{gathered}
\left|\operatorname{Im}\left(\alpha^{-}\right)\right|=\left\{\left(\begin{array}{cc}
1 & 2 \\
-1 & -1
\end{array}\right),\left(\begin{array}{cc}
1 & 2 \\
-1 & -2
\end{array}\right),\left(\begin{array}{cc}
1 & 2 \\
-2 & 1
\end{array}\right),\left(\begin{array}{cc}
1 & 2 \\
-2 & -2
\end{array}\right)\right\}=4 \\
\left|\operatorname{Im}\left(\alpha^{*}\right)\right|=\left\{\begin{array}{cc}
\left(\begin{array}{cc}
1 & 2 \\
1 & -1
\end{array}\right),\left(\begin{array}{cc}
1 & 2 \\
-1 & 1
\end{array}\right),\left(\begin{array}{cc}
1 & 2 \\
1 & -2
\end{array}\right),\left(\begin{array}{cc}
1 & 2 \\
-1 & 2
\end{array}\right), \\
\left(\begin{array}{cc}
1 & 2 \\
2 & -1
\end{array}\right),\left(\begin{array}{cc}
1 & 2 \\
-2 & 1
\end{array}\right),\left(\begin{array}{cc}
1 & 2 \\
2 & -2
\end{array}\right),\left(\begin{array}{cc}
1 & 2 \\
-2 & 2
\end{array}\right),
\end{array}\right\}=4
\end{gathered}
$$

Table 2.2: Values of elements in $P T S_{n}$

\begin{tabular}{|c|c|c|c|}
\hline$n$ & $\left|\operatorname{Im}\left(\alpha^{-}\right)\right|$ & $\left|\operatorname{Im}\left(\alpha^{*}\right)\right|$ & $\left|P S T_{n}\right|=n^{n}+n^{n}\left(2^{n}-2\right)$ \\
\hline 1 & 1 & - & 1 \\
\hline 2 & 4 & 8 & 12 \\
\hline 3 & 27 & 162 & 189 \\
\hline 4 & 256 & 3584 & 3840 \\
\hline 5 & 3125 & 93750 & 96875 \\
\hline
\end{tabular}

Polarity of elements in SSing ${ }_{n}$ is as follows:

When $n=1$

$$
\left|P \operatorname{Sing}_{1}\right|=0
$$

When $n=2$

$$
\left|P S \operatorname{Sing}_{2}\right|=\left\{\left(\begin{array}{cc}
1 & 2 \\
-1 & -1
\end{array}\right),\left(\begin{array}{cc}
1 & 2 \\
-1 & 1
\end{array}\right),\left(\begin{array}{cc}
1 & 2 \\
1 & -1
\end{array}\right),\left(\begin{array}{cc}
1 & 2 \\
-2 & -2
\end{array}\right),\left(\begin{array}{cc}
1 & 2 \\
-2 & 2
\end{array}\right),\left(\begin{array}{cc}
1 & 2 \\
2 & -2
\end{array}\right)\right\}=6
$$

Image of element in PSSing 2

$$
\begin{gathered}
\left|\operatorname{Im}\left(\alpha^{-}\right)\right|=\left\{\left(\begin{array}{cc}
1 & 2 \\
-1 & -1
\end{array}\right),\left(\begin{array}{cc}
1 & 2 \\
-2 & -2
\end{array}\right)\right\}=2 \\
\left|\operatorname{Im}\left(\alpha^{*}\right)\right|=\left\{\left(\begin{array}{cc}
1 & 2 \\
-1 & 1
\end{array}\right),\left(\begin{array}{cc}
1 & 2 \\
1 & -1
\end{array}\right),\left(\begin{array}{cc}
1 & 2 \\
-2 & 2
\end{array}\right),\left(\begin{array}{cc}
1 & 2 \\
2 & -2
\end{array}\right)\right\}=4
\end{gathered}
$$


Table 2.3: Values of elements in PSSing $_{n}$

\section{Main results}

\begin{tabular}{|c|c|c|c|}
\hline$n$ & $\left|\operatorname{Im}\left(\alpha^{-}\right)\right|$ & $\left|\operatorname{Im}\left(\alpha^{*}\right)\right|$ & $\left|P \operatorname{Sing}_{n}\right|=\left(2^{n}-n\right)\left(n^{n}-n !\right)$ \\
\hline 1 & 0 & 0 & 0 \\
\hline 2 & 2 & 4 & 6 \\
\hline 3 & 21 & 126 & 3712 \\
\hline 4 & 229 & 3254 & 96160 \\
\hline 5 & 3125 & 89910 & \\
\hline
\end{tabular}

\section{Theorem 3.1}

Let $S=P S S_{n}$ and if $\alpha \in P S S_{n}$ then $\left|P S S_{n}\right|=n !+\left(2^{n}-n\right) n !$

Proof

Let $X_{n}=\{1,2,3, \cdots, n\}, X_{n}^{*}=\{-n, \cdots,-3,-2 .-1.0,1,2,3, \cdots, n\}$ and $X_{n} \subset X_{n}^{*}$. It follows from counting argument that there are $n$ ! numbers of elements for $\left|\operatorname{Im}\left(\alpha^{-}\right)\right|$. Since the $|\operatorname{Im}(\alpha)|$ is either $+i$ or $-i$ where $i=$ $1,2,3, \ldots, n$ and by binomial theorem for a positive $n$ where $\sum_{k=0}^{n}\left(\begin{array}{l}n \\ k\end{array}\right)=2^{n}$, the $\left|\operatorname{Im}\left(\alpha^{*}\right)\right|$ in $P S S_{n}$ have $(n-1)$ groups and each group consists of $\left(2^{n}-n\right) n$ ! elements. Multiply and summing we have $n !+\left(2^{n}-n\right) n$ ! elements and this is equivalent to $2^{n} n !-n$ !

\section{Theorem 3.2}

Let $S=P S T_{n}$ and if $\alpha \in P S T_{n}$ then $\left|P S T_{n}\right|=n^{n}+n^{n}\left(2^{n}-2\right)$

Proof

Let $\alpha: X_{n} \rightarrow X_{n}^{*}$ and $\alpha(i)= \pm j$ where $i \in \operatorname{dom}(\alpha)$ and $\operatorname{lm}(\alpha) \subset X_{n}^{*}$. If the $|\operatorname{Im}(\alpha)|$ is either $i$ or $-i$ for $i=$ $1,2,3, \ldots, n$ then the nature of $\left|\operatorname{Im}\left(\alpha^{*}\right)\right|$ is such that each group consists $n^{n}\left(2^{n}-2\right)$ elements. Since the semigroup is $1-1$ mapping then $|\alpha S|=n^{n}$ when $\left|\operatorname{Im}\left(\alpha^{*}\right)\right|$ for each $n$. Hence by summing $\left|P S T_{n}\right|=n^{n}+$ $n^{n}\left(2^{n}-2\right)$.

\section{Theorem 3.3}

Let $S=P \operatorname{SSing}_{n}$ and if $\alpha \in P S \operatorname{Sing}_{n}$, then $\left|P S \operatorname{Sing}_{n}\right|=\left(2^{n}-1\right)\left(n^{n}-n !\right)$

Proof

Let $\alpha \in P \operatorname{SSing}_{n}$ and such that $\alpha: \operatorname{dom}(\alpha) \subseteq X_{n} \rightarrow \operatorname{Im}(\alpha) \subset X_{n}^{*}$ and from theorem 1.3 it follows that $\left|\operatorname{SSing}_{n}\right|=2^{n}\left(n^{n}-n !\right)$ and $\left|\operatorname{Im}\left(\alpha^{+}\right)\right|=n^{n}-n$ ! and also there are $n$ such elements having the property $\left|\operatorname{Im}\left(\alpha^{+}\right)\right|=\left|\operatorname{Im}\left(\alpha^{-}\right)\right|=n^{n}-n !$... equation (3.1) Thus, from table 2.3 and the $\left|\operatorname{Im}\left(\alpha^{*}\right)\right|$ is simplified as followed:

$$
\begin{aligned}
& 2^{n}\left(n^{n}-n !\right)-2\left(n^{n}-n !\right) \\
& 2^{n} n^{n}-2^{n} n !-2 n^{n}-2 n ! \\
& 2^{n} n^{n}-2 n^{n}-2^{n} n !-2 n ! \\
& n^{n}\left(2^{n}-2\right)-n !\left(2^{n}-2\right) \\
& \left(2^{n}-2\right)\left(n^{n}-n !\right) \ldots \ldots \text { equation (3.2). }
\end{aligned}
$$

thus combining equation (3.1) and equation (3.2) yields $\left|P S \operatorname{Sing}_{n}\right|$ for each $n$ 


$$
\begin{gathered}
\left(n^{n}-n !\right)+\left(2^{n}-2\right)\left(n^{n}-n !\right) \\
\left(n^{n}-n !\right)\left[1+\left(2^{n}-2\right)\right] \\
\left(n^{n}-n !\right)\left(1+2^{n}-2\right) \\
\left(2^{n}-1\right)\left(n^{n}-n !\right)
\end{gathered}
$$

and hence the result $\left|P S S_{i n g}\right|=\left(2^{n}-1\right)\left(n^{n}-n !\right)$

\section{Summary of results}

The following results with sequences were obtained for all $n$

Let $S=P S S_{n}$ then $\left|P S S_{n}\right|=n !+\left(2^{n}-n\right) n !$ which generates the sequence $1,6,42,360,3420,45360, \ldots$

Let $S=P S T_{n}$ then $\left|P S T_{n}\right|=n^{n}+n^{n}\left(2^{n}-2\right)$ which generate the sequence $1,12,189,3842,96875, \ldots$

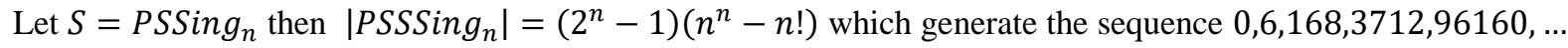

\section{Conclusion}

It is hereby recommended that the idempotent, chain decomposition of polarity of full and partial transformation can also be study.

\section{References}

Adeniji, A.O. (2012)," Identity difference transformation semigroups",PhD Thesis, submitted to Department of Mathematics, University of Ilorin, Ilorin, Kwara state Nigeria.

Bashar, A. (2010), "Combinatorial properties of the alternating and dihedral and homomorphic images of Fibonacci groups". PhDThesis, submitted to Department of Mathematics, University of Jos, Nigeria.

Adeshola, D.A. (2013), “Some semigroups of full contraction mappings of a finite chain", PhD Thesis, submitted to Department of Mathematics, University of Ilorin, Ilorin, Kwara state Nigeria.

Howie, J.M. (1995), "Fundamentals of semigroup Theory, London Math. Soc. Monographs”, New series, 12 Oxford Science Publications. The Claredon Press, Oxford University Press, New York.

Bakare, G.N. (2015), “Some results on properties of alternating semigroups",Nigerian Jounal of Mathematics and Application 24, $184-192$.

Howie, J.M. (2002), “Semigroups, past, present and future”,Proceedings of the international Conference in Algebra and its Apllication, 6 - 21.

Laradji, A. and Umar, A. (2004), "Combinatorial results for semigroups of order - preserving partial transformations",Jounal of algebra 278, 342 - 359.

Howie, J.M. (1995), "Fundamentals of semigroup Theory”, London Math.Soc. Monographs, New series, 12 Oxford Science Publications. The Claredon Press, Oxford University Press, New York.

Mogbonju, M.M. (2015), “Some combinatoric properties of signed transformation semigroups", PhD Thesis submitted to Department of Mathematics, University of Ilorin, Kwara state, Nigeria.

Mogbonju, M.M., and Ogunleke, A.I. (2019), "Polarity in signed order - preserving and order - decreasing semigroup".International Journal of Pure Algebra"9(5).

Umar, A. (1992), “On the semigroup of order - decreasing finite full transformations",Proceeding of the Royal $S$ Society of Edinburg, 120A, 129 - 142.

Howie, J.M. (1966), "The subsemigroupgeneratedby the idempotents of a full transformation semigroup", $J$. London Maths. Sec., 41, 707 - 716. 